\title{
Agôn
}

9| 2021

Rater

\section{L'envers du décor. Ragnar Kjartansson et les clichés}

Hélisenne Lestringant

\section{(2) OpenEdition}

Journals

Édition électronique

URL : https://journals.openedition.org/agon/8434

DOI : 10.4000 /agon.8434

ISSN : 1961-8581

\section{Éditeur}

Association Agôn

\section{Référence électronique}

Hélisenne Lestringant, «L'envers du décor. Ragnar Kjartansson et les clichés », Agôn [En ligne], 9 | 2021, mis en ligne le 07 octobre 2021, consulté le 18 janvier 2022. URL : http:// journals.openedition.org/agon/8434; DOI : https://doi.org/10.4000/agon.8434

Ce document a été généré automatiquement le 18 janvier 2022.

Association Agôn et les auteurs des articles 


\title{
L'envers du décor. Ragnar Kjartansson et les clichés
}

\author{
Hélisenne Lestringant
}

\section{Du cliché}

1 Un cœur très rouge percé d'une flèche, un papillon pour la joie, un homme qui regarde sa montre parce qu'il attend, voilà autant d'images clichées dont regorge notre imaginaire collectif. D'une représentation non questionnée de la réalité découlent une attitude et une conduite à adopter pour signifier des états ou des actions. Correspondant d'abord à un terme photographique, le cliché renvoie à la surface d'une plaque métallique en relief établie par moulage en vue d'une impression typographique reproductible à l'infini, avant de signifier une «idée ou expression toute faite trop souvent utilisée ${ }^{1} »$. Le terme de cliché, dans son acception élargie, garde la connotation d'un élément extérieur, existant d'abord comme une image lointaine, remarquable par son aspect esthétique, voire plastique. Or, les clichés passent pour dangereux, notamment en interprétation théâtrale ${ }^{2}$.

2 Peu reluisants, ils n'ont pas leurs lettres de noblesse en études théâtrales, grands absents des dictionnaires de Patrice Pavis ou de Michel Corvin par exemple ${ }^{3}$, prouvant qu'ils sont associés au rater, marge sombre du théâtre à écarter de son Histoire officielle. Plus rarement, le cliché est envisagé comme une inspiration possible pour une interprétation dramatique fine. Réfléchir à ses vertus pour le jeu reviendrait à ne pas commencer par la recherche d'authenticité mais par l'aspect purement extérieur, avant de dépasser le poncif pour en tirer une vérité.

Les clichés, par leur richesse esthétique paradoxale, intéressent de près l'artiste plasticien, musicien et performeur islandais Ragnar Kjartansson, afin de montrer les rouages mal huilés de notre société contemporaine. Jouant avec les clichés qu'il associe au théâtre, il a développé, depuis ses années de formation à l'Académie des Arts d'Islande, à l'encontre de l'enseignement incitant à développer des images uniques de la réalité, un univers pictural et plastique de carton-pâte, pour créer dans des films et 
des performances, des décors et des situations de jeu romantiques et émouvants. Dans ses performances, il développe une esthétique et une dramaturgie du rater : une action scénique se répète sans évoluer dans un dispositif dont les acteurs sont prisonniers.

\section{Le cliché chez Ragnar Kjartansson}

4 Né en 1976 d'une mère actrice et d'un père réalisateur, élevé dans les images et les spectacles, Ragnar Kjartansson explique par sa mythologie personnelle sa fascination pour l'envers du décor au théâtre, qu'il observait, enfant, depuis les coulisses. Entre l'envers et l'endroit, aujourd'hui encore, il ne peut trancher lequel est le plus sublime, des décors romantiques peints avec art, ou des contrepoids et panneaux de bois nus, numérotés. Fasciné par l'apparence la plus extérieure, clichée et glamour du théâtre et du monde, il ne cesse de mettre en scène, dans ses films, ses peintures et ses performances, des dispositifs bloqués qui soulignent le cliché par la répétition. En même temps, il célèbre avec humour leur envers, visible, par exemple par un coin de toile à peine achevé. Cet envers, montré alors qu'il devrait rester caché, renvoie métaphoriquement aux simulacres du monde occidental contemporain.

Chez Ragnar Kjartansson, le cliché s'incarne en particulier dans une esthétique du quotidien. L'artiste se concentre sur des actions anodines, répétées plusieurs heures jusqu'à se dénaturer et devenir étranges. Si Jean Genet décelait dans la cérémonie de la messe le plus grand spectacle théâtral, Ragnar Kjartansson s'ingénie, lui, à ressaisir dans différents cadres familiers assez banals ce qui relève du théâtre. Il peut s'agir d'une rencontre entre une femme et un dépanneur venu réparer sa machine à laver (Take me by the dishwasher: Memorial for a Marriage, 2011-2014) ou d'un concert réalisé par un crooneur des années 1960 (God, 2007). La théâtralité des moments du quotidien, selon la définition de Nikolai Evreinov, le fascine car elle dévoile les lois régissant « la transformation créative du monde que nous percevons ${ }^{4} »$.

6 Célèbre pour ses œuvres picturales et filmiques, l'artiste islandais s'impose aussi dans le monde de l'art contemporain par des œuvres scéniques dans lesquelles il joue parfois. Dans les villes accueillant ses expositions, il se produit souvent en concert avec son groupe « Ragnar Kjartansson and the All Star Band » mais il s'impose comme véritable «metteur en scène ${ }^{5}$ » avant tout par des saynètes au sein de ses expositions. Les clichés y sont représentés en chair et en os, par des comédiens ou par l'artiste lui-même, lors d'une scène de quelques minutes se répétant durant plusieurs heures. Dans la réitération à l'infini se construit une esthétique du rater que l'artiste décline d'une œuvre à l'autre.

\section{Rater et répétition chez Ragnar Kjartansson}

7 Trois œuvres performatives de Ragnar Kjartansson seront abordées ici : Bonjour au Palais de Tokyo en 2015, Guerre (Krieg) en 2016 et Salon cru : un jeu cru (Raw Salon : ein Rohspiel) en 2017 au théâtre de la Volksbühne am Rosa-Luxemburg Platz à Berlin. Pour chacune de ces performances, l'artiste développa une esthétique du rater, mais il finit par un échec dans le dernier projet. En effet, le dernier spectacle se solda par un ratage définitif, presque tragique, manquant le but visé et que l'on peut donc qualifier d'échec. Pour chacun des spectacles, l'artiste dessina et peignit le décor avec son équipe, créant des cadres parfaitement adaptés à chaque scène clichée qui devait s'y jouer. 
Ces spectacles sont qualifiés de performatifs puisque chacune de leur répétition variait en fonction de la "co-présence entre des acteurs et des spectateurs ${ }^{6}$ ". Même si le metteur en scène visait l'identité de la scène répétée d'une fois sur l'autre dans Bonjour et Salon cru : un jeu cru, celle-ci variait au fil de ses répétitions en fonction des acteurs et du public, un peu pour le premier projet, beaucoup pour le deuxième. La nomenclature d'Erika Fischer-Lichte se trouve justifiée par les méthodes de jeu de l'acteur employées au sein des performances. Ragnar Kjartansson, déclarant le premier ne pas pouvoir jouer quelqu'un d'autre que lui-même, marque son appartenance à la catégorie du "performeur ", utilisant son corps réel comme corps scénique. En 2009, pendant toute la durée de la Biennale de Venise, il s'est ainsi joué dans le rôle de l'artiste peintre au travail, se remettant inlassablement chaque jour à son chevalet dans la nature italienne. De même, quand il embauchait des acteurs pour jouer dans ses performances, il se contentait de les guider lors de quelques rares répétitions, et les incitait ensuite à se faire confiance à partir du parcours simple fixé à travers le décor ${ }^{7}$.

Ragnar Kjartansson confond volontairement les différentes catégories artistiques dont il s'empare, expliquant: "avec la répétition, les éléments narratifs tels que les chansons, les concerts, les opéras peuvent perdre leur forme traditionnelle et devenir statiques - mais vibrants, comme des tableaux ou des sculptures; je regarde souvent mes performances comme des sculptures et les vidéos comme des tableaux ${ }^{8} »$. Les scènes étudiées déjouent, par le rater d'une action - la rencontre amoureuse dans Bonjour, la mort dans Guerre, l'évolution du dialogue dans Salon cru: un jeu cru - les codes du drame et les attentes des spectateurs, pensées en fait comme de véritables tableaux vivants. À force d'être répété, le rater de l'action au cœur de la performance dénature le sens de la scène jouée, et conduit les acteurs à se questionner sur leur place d'interprète au cœur de ces formes hybrides, entre théâtre et art plastique. Les mécanismes du rater seront exposés dans les trois œuvres ici envisagées ; une attention particulière visera la dernière mise en scène et son débordement du rater vers l'échec.

Quelques remarques s'imposent sur la localisation des trois œuvres, tant sur leurs sites d'accueil que sur les lieux représentés. Entre la France et l'Allemagne, la place réservée aux œuvres de Ragnar Kjartansson varie. Tandis que la première œuvre, Bonjour, fut accueillie au Palais de Tokyo pour la première exposition personnelle de l'artiste en France en 2015, les deux autres se déroulèrent au théâtre de la Volksbühne à Berlin, en 2016 et 2017, où l'artiste avait déjà produit un opéra sans acteurs. Cette différence de lieux est due aux différentes réceptions du genre de la performance de part et d'autre du Rhin. Dans les œuvres elles-mêmes, le décor inspiré par le cadre français pour Bonjour reproduisait l'image d'Épinal d'une place de village des années 1950, tandis qu'en Allemagne, la pièce Guerre reconstituait un champ de bataille prussien des siècles passés, et Salon cru : un jeu cru un salon bourgeois. Ragnar Kjartansson s'appliquait à décliner les clichés propres aux différents pays et pariait sur la complicité des spectateurs locaux pour assurer la réussite du rater.

\section{Bonjour ou le rater de la rencontre}

Ce média ne peut être affiché ici. Veuillez vous reporter à l'édition en ligne http:// 
L'exposition au Palais de Tokyo, "Seul celui qui connaît le désir ", d'octobre 2015 à janvier 2016, introduisait les spectateurs dans un monde plein de désirs et de frustrations, sous le haut patronage de Goethe. Le titre était un écho à l'un de ses poèmes évoquant « l'aspiration éternellement inassouvi[e ${ }^{\natural}$ ». De fait, l'élan amoureux mais l'impossibilité de la communication étaient déclinés dans plusieurs œuvres ; dans la série de performances filmées "Scenes from Western Culture », un couple en tenue de soirée dînait sans échanger une parole dans un restaurant luxueux. Le décorum parfaitement romantique mais figé et étouffant était ainsi mis à nu face à la caméra, seul témoin de la théâtralité de la scène. La performance Bonjour présentait elle aussi un cadre idyllique, aboutissant à la seule déception du spectateur face à l'échec d'une rencontre. Pourtant, lorsque l'observateur comprenait que la rencontre n'aurait jamais lieu, selon l'éternel retour du "rater », la déception, muée en frustration programmée à l'infini, devenait jubilatoire.

Dans cette exposition parisienne, Ragnar Kjartansson revenait, par différents supports, à l'un de ses sujets de prédilection, l'envers du décor. Il présentait notamment une série de rochers de théâtre, peints sur des paravents de bois, entre et derrière lesquels les spectateurs pouvaient circuler, découvrant la structure nue du décor qui ne faisait aucunement illusion. Lors d'un entretien avec le commissaire d'exposition Julien Fronsacq, l'artiste expliquait :

Les falaises installées au Palais de Tokyo sont, d'un côté, un paysage, de l'autre, seulement du contreplaqué. J'ai travaillé comme technicien au théâtre et les coulisses sont l'endroit que je préfère. Le décor a deux faces, un avant et un arrière. Il est à la fois sublime et banal et pose la question de savoir ce qui est quoi ${ }^{10}$.

Coupe de l'installation de Bonjour, depuis la mezzanine du $1^{\text {er }}$ étage du musée.

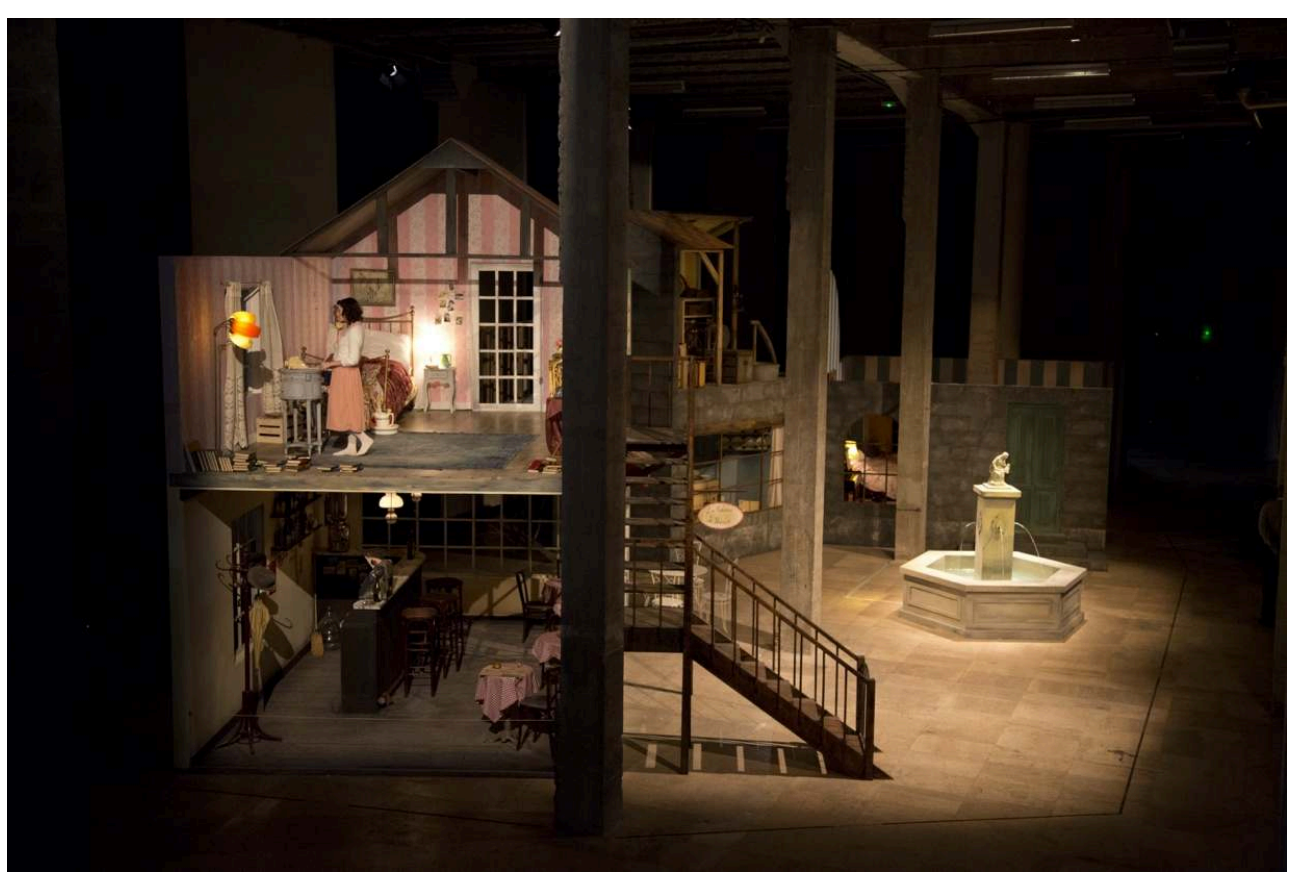

(c) Luhring Augustine, New York \& i8 Gallery, Reykjavik. Photo : Justine Emard.

La performance Bonjour reproduisait cette mise à nu de l'illusion, entre sublime et banal, avec une saynète de 7 minutes répétée en boucle pendant 6 heures. Le décor, dessiné et peint par l'artiste, reproduisait une place de village idyllique de la France des 
années 1950, au centre de laquelle trônait une fontaine encadrée par deux maisons à l'échelle 1 . De style très réaliste, il était peaufiné dans ses moindres détails, jusqu'à certains objets d'époque mis en évidence, comme un ancien numéro de L'Humanité posé sur une table. Les deux habitations ressemblaient à des maisons de poupées, du côté de la femme, les murs étaient tapissés d'un papier peint strié de rose, et de bleu du côté de l'homme. La femme logeait au-dessus d'un bar, écoutait Charles Trenet sur un gramophone, tandis que l'homme au rez-de-chaussée de sa maison, enfonçait quelques notes sur son piano orné d'un buste de Beethoven; autant d'éléments qui soulignaient une différence d'appartenance sociale. Le décor oscillait entre différents codes de représentation, dénonçant son illusion à la Brecht mais obéissant aux règles de l'hyperréalisme du moindre accessoire, proche du naturalisme défini par André Antoine, offrant l'observation d'une «tranche de vie » quand un pan de mur abattu permettait d'épier le quotidien le plus intime de deux personnes. Les visiteurs de l'exposition pouvaient circuler autour de l'ensemble constitué par les maisons et la fontaine, mais, pour ne pas gêner les performeurs, ne pouvaient entrer dans le décor.

Du côté de l'homme (Vincent Gaudin).

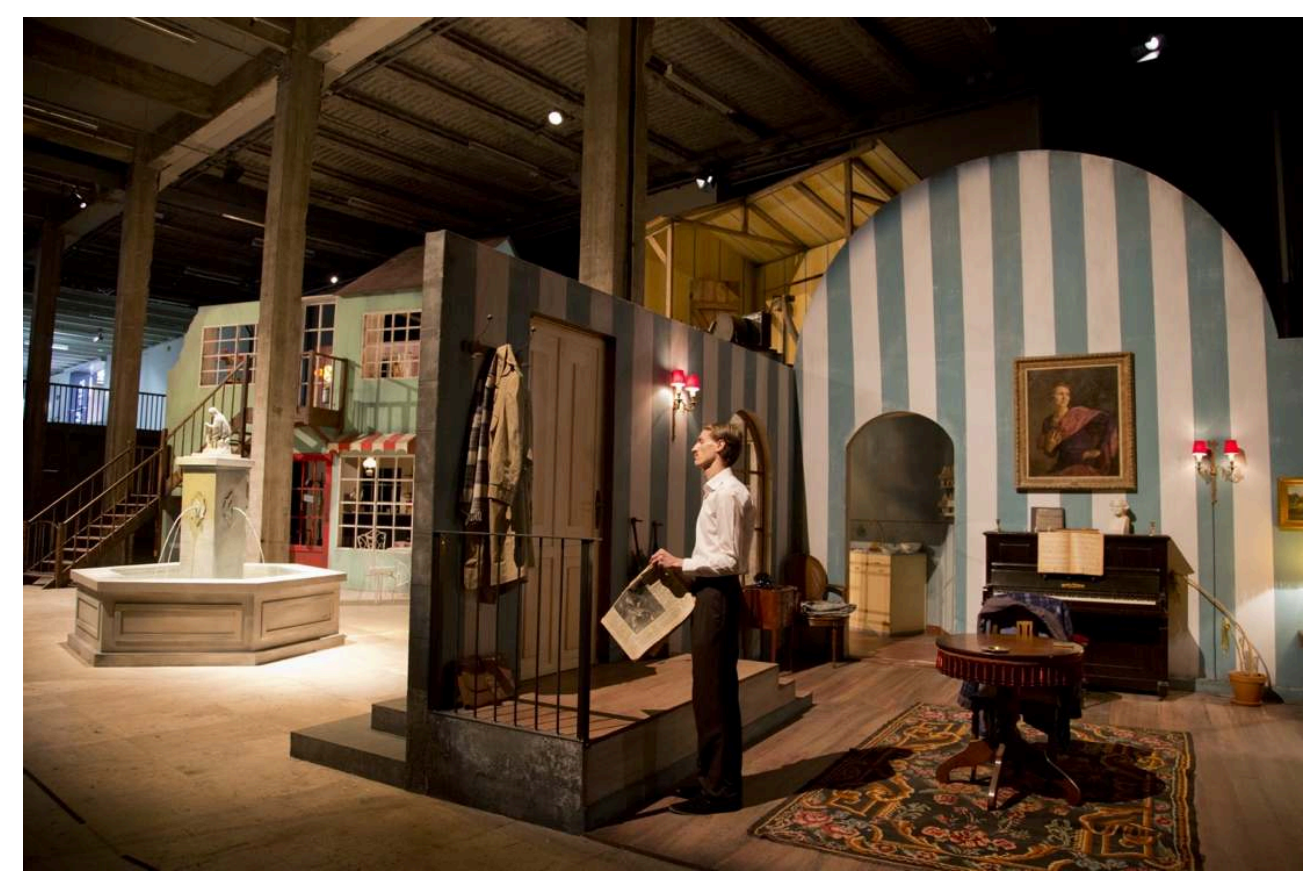

(c) Luhring Augustine, New York \& i8 Gallery, Reykjavik. Photo : Justine Emard.

\section{Structure dramaturgique}

La dramaturgie reposait sur deux routines, vécues dans chaque maison par l'homme et la femme, puis sur leur rencontre à la fontaine. La femme se réveillait déjà vêtue dans son lit, s'étirait, mettait le disque de la chanson «La Mer » de Charles Trenet, nouait quelques boutons du haut sa robe, enfilait sensuellement ses bas de coton, se maquillait de manière non réaliste, effleurant ses poudres, regardait les fleurs dans son vase, décidait qu'il leur manquait de l'eau au moment où la chanson de Charles Trenet d'un peu moins de 4 minutes s'achevait. Elle ouvrait alors sa porte, descendait la volée de marches du perron et rencontrait son voisin à la fontaine. De son côté, l'homme se 
réveillait, s'étirait, passant dans sa cuisine, il se versait une tasse de café, lisait le journal, jouait quelques notes au piano, allumait sa pipe puis sortait pour rencontrer, à la fontaine, sa voisine.

Ils se regardaient, il lui disait « Bonjour », elle lui répondait « Bonjour ». La femme ôtait les fleurs du vase qu'elle plongeait dans l'eau et avant que la situation n'ait pu évoluer, elle remontait chez elle. Sur le pas de sa porte, elle jetait un dernier regard à son voisin qui la regardait encore, puis elle rentrait chez elle, défaisait quelques boutons du haut de sa robe, et se couchait dans son lit.

De son côté, l'homme, après avoir regardé sa voisine disparaître, tirait quelques bouffées sur sa pipe, puis rentrait chez lui pour se mettre au lit. La lumière descendait doucement avant de revenir au pépiement d'oiseaux. La femme et l'homme se réveillaient, bientôt la chanson de Charles Trenet résonnait, pour tout recommencer. La rencontre étant bloquée chaque fois au seuil du simple salut, la bascule dramatique n'avait jamais lieu. Le sort des personnages condamnés à se dire "Bonjour " était tragiquement et presque ironiquement annoncé dès le titre de la performance, prouvant a posteriori aux spectateurs qu'ils étaient seuls responsables de leurs attentes déçues.

\section{Organisation logistique}

18 Durant l'exposition au Palais de Tokyo, la performance était visible aux horaires d'ouverture du musée, de midi à minuit, au même titre qu'une œuvre picturale. Par blocs de 6 heures, les comédiens alternaient en binômes, avec une pause de 20 minutes au cœur d'une session ${ }^{11}$. Les performeurs, formés en art dramatique ou en danse, étaient au nombre de huit, quatre jeunes femmes et quatre jeunes hommes. Je fus l'une des quatre performeuses, aux côtés de Margherita Bertoli, Héloïse Lesimple, Léa Pérat, Yoann Piquet, Guillaume Mivekannin, Vincent Gaudin, Arnaud Guy. Cette perspective depuis l'intérieur du dispositif sera mise à profit pour une réflexion sur le rater du côté de l'interprétation dramatique.

19 Lors du vernissage, la boucle initiale, d'une dizaine de minutes, fut présentée : la femme et l'homme se déshabillaient de manière réaliste, la femme, par exemple, enlevait son jupon et passait une chemise de nuit avant de se coucher. Après le vernissage, Ragnar Kjartansson décida de procéder à «des changements dramatiques»: il réduisit la boucle, passant d'une dizaine de minutes à environ 7 minutes, afin que le spectateur comprenne facilement que l'itération faisait partie de l'œuvre. L'homme et la femme devaient désormais signifier le déshabillement plus qu'ils ne le jouaient, le jupon comme la chemise de nuit de la femme restèrent sur leurs cintres en loge.

20 L'artiste précisa aussi ses indications de jeu : «Bonjour » devait être dit de manière non théâtrale, très quotidienne, la parole ne devant pas être projetée. Le mot devait refléter la possibilité de la rencontre qui n'était pas spectaculaire mais proche de notre expérience de tous les jours. La "superproduction" du décor, pour reprendre les termes de l'artiste, servait un contact infime, visuel et verbal, entre les deux personnages.

21 Le metteur en scène ne demandait pas aux performeurs un type de jeu du «moment présent » dans l'espace-temps ténu de la représentation, essentiel dans la performance theory de Richard Schechner, mais au contraire une chorégraphie invariable malgré l'installation. Dès lors, le registre d'interprétation induit par la performance semblait 
extraire performeurs et spectateurs du paradigme des arts "mourants " de la scène, dépeints par Heiner Müller ${ }^{12}$. En effet, les maisons de poupée semblaient habitées par les figurines d'une boîte à musique sur les notes de Charles Trenet. La performance définissait alors justement sa qualité par l'effort des comédiens à ne rien laisser paraitre de leur lassitude humaine de « mourant » malgré la répétition.

Ragnar Kjartansson est à plusieurs égards héritier des démarches des performeurs des années 1970. Dans ce cas précis, l'insistance sur les clichés, rendue par la répétition sur la durée, était associée à la notion d'épreuve, tant du côté du performeur que du spectateur:

Je crois que la différence entre mon approche et celle de la tradition classique de la performance [...] est une question d'attitude et de contexte. [...] Il est extrêmement satisfaisant de réaliser quelque chose qui a l'air d'une prouesse. Mais les miennes sont des prouesses de spectacle. Si certaines de mes performances nécessitent un effort physique éprouvant, la difficulté principale reste l'ennui et le sentiment d'insignifiance ${ }^{13}$.

Ragnar Kjartansson nous remercia personnellement le soir du vernissage au Palais de Tokyo, puis à la toute fin de l'exposition; entre ces deux moments, nous avions compris dans quelle mesure le terme d'« épreuve » convenait à cette expérience de spectacle vivant.

Pour certains performeurs, ce type de jeu était aisé, le circuit à accomplir se déroulait tel une chorégraphie automatique dans un refuge fictionnel hors de la vraie vie, dans l'espace calfeutré du musée, permettant même une introspection de l'ordre du songe, sous l'effet d'un mantra. D'autres performeurs étaient au contraire usés par la répétition qui leur faisait perdre le sens de l'interprétation théâtrale.

En effet, les enjeux restant les mêmes d'une boucle à l'autre, le risque de rater était presque nul ; le comédien était alors réduit à un enchaînement de gestes. Il achevait sa partition solitaire pour dire "Bonjour " au partenaire, entrevoyait enfin la possibilité d'une situation de jeu à deux, mais cette perspective s'effaçait aussitôt comme un mirage, et il revenait à sa solitude. Différentes couches de rater émergeaient: la rencontre ratait du fait de la répétition qui à son tour empêchait l'acteur de rater son rôle. Certains acteurs vécurent cette expérience comme un échec personnel.

Employés pour jouer sur des créneaux de six heures rappelant des horaires de bureau, les performeurs développaient un rapport inhabituel à leur pratique. En 2013, Ragnar Kjartansson s'était déjà amusé de cet aspect avec le groupe The National auquel il avait demandé de jouer sa chanson $A$ Lot of Sorrow "en boucle pendant six heures ${ }^{14}$ ». Le morceau, émergeant normalement au sein d'un concert comme une pièce maîtresse et unique, devenait ici sériel. Ragnar Kjartansson s'amusait à abîmer le mythe de l'inspiration artistique : ce n'est pas elle qui menait The National à entonner soudain sa chanson émouvante mais la seule partition pré-écrite. De même, dans Bonjour, la rencontre amoureuse n'était pas due à l'inspiration des acteurs, mais fixée dans un schéma d'actions chronométrées.

27 Par la fragmentation d'une œuvre qui aurait pu être plus vaste, Ragnar Kjartansson donnait une autre portée aux gestes artistiques. Esseulée, la chanson de The National n'avait plus la même émotion, tout comme dans Bonjour les comédiens ne jouaient pas le reste d'un drame amoureux dont la rencontre à la fontaine aurait été la prémisse. En accomplissant toujours le même fragment de leur art, les interprètes étaient mis au supplice comme Sisyphe faisant rouler son rocher. Alors qu'ils prêtaient leurs corps à 
l'interprétation d'une scène ou d'une chanson, le dispositif transformait leur élan artistique en un geste cliché automatisé comme un GIF (Graphics Interchange Format). En révélant que la pulsion artistique était reproductible, Ragnar Kjartansson interrogeait les interactions humaines pour rencontrer l'autre, tomber amoureux, discuter ou mourir.

Un jour, à la fin du projet, juste avant l'ouverture du musée, avec la complicité du personnel de l'exposition, nous nous sommes retrouvés avec les performeurs de Bonjour pour réaliser un film libérateur. Dans nos costumes et le décor, nous avons enfin joué les scènes proscrites de la douce chorégraphie habituelle. Dans une séquence, Margherita Bertoli, dans la chambre de la femme, se déchaînait comme dans un concert de hard rock. À la fontaine, les quatre femmes adressaient leur «Bonjour " aux quatre hommes qui répondaient en chœur. Le film permit d'expurger les frustrations et de rompre l'isolement de chaque acteur au cours de la performance. Ragnar Kjartansson fut ravi de cette revanche, ce qui nous conforta dans l'idée que la cage de verre du dispositif avait secrètement poussé à cultiver les envies de jeu inassouvies.

\section{Les pièces à la Volksbühne}

La Volksbühne, scène emblématique de Berlin Est, entretenait, sous la direction du metteur en scène Frank Castorf jusqu'en 2017, un étroit lien avec le rater et l'échec. Une anecdote l'illustre: le directeur qui accueillit en 1993 le jeune Christoph Schlingensief alors qu'il n'était encore connu que comme réalisateur de films underground sanglants et n'avait aucune expérience en mise en scène, lui avait déclaré qu'il était autorisé à « rater » à la seule condition de « rater alors complètement ${ }^{15} »^{16}$. Cette scène prêchait les formes de théâtre qu'Hans-Thies Lehmann avait voulu regrouper sous le terme de "théâtre postdramatique " à la fin des années $1990^{17}$. Ce genre se caractérisait comme " le premier à expliciter le réel comme "co-acteur " permanent au niveau pragmatique (et pas seulement théorique)». La Volksbühne contribua à ce que "[1]'irruption du réel dev[ienne] l'objet, pas seulement de la réflexion [...] mais de l'agencement théâtral même ${ }^{18}$ ». Cette scène subventionnée proposait ainsi des expériences particulières à ses spectateurs, généralement ouverts aux pièces de 6 heures d'un Frank Castorf ou à celles particulièrement physiques d'un Herbert Fritsch. Dans cet esprit, les comédiens de l'Ensemble - désignant la troupe attachée au théâtre national allemand - étaient habitués à prendre des risques sur scène, loin des définitions habituelles de ratage, décrites par Hans-Thies Lehmann :

Même s'il lui demeure indiscutablement attaché, le réel au théâtre fut toujours écarté pour des raisons esthétiques ou conceptuelles. Généralement, ce ne sont que les « pannes » qui l'illustrent avec le plus d'éclat. Au-delà de ce traumatisme et de cette image idéale que l'on projette dans le théâtre, l'irruption du réel dans le jeu scénique, on le perçoit généralement seulement sous la forme d'erreurs embarrassantes que relatent les anecdotes et les blagues dont regorge la vie du théâtre ${ }^{19}$.

30 Sur cette scène, fleuron de l'avant-garde théâtrale favorable à l'irruption du réel et de l'accidentel, Ragnar Kjartansson avait, dès 2014, présenté une pièce sans acteur, Le Son de la révélation du divin (Der Klang der Offenbarung des Göttlichen), pour laquelle il avait collaboré avec l'orchestre de Babelsberg, comme pour son projet suivant, Guerre. 


\section{Guerre (Krieg, 2016)}

Dans ce spectacle d'une heure présenté comme un opéra en un acte pour un acteur, le spectateur assistait, après la déroute d'une armée, à la longue et pénible agonie d'un soldat dans un décor de champ de bataille peint aux couleurs romantiques d'un coucher de soleil flamboyant. Son costume jaune vif à veste bleue était celui d'un soldat prussien. L'acteur Maximilian Brauer, seul en scène, se recroquevillait, se redressait, pleurait, poussait de légères plaintes, des gémissements, des râles, quelques cris. Il ne prononçait pas un seul mot. L'opéra en un acte de Kjartan Sveinsson, membre du groupe post-rock Sigur Rós, l'accompagnait tout du long, selon des variations de volumes. Les quelques répétitions s'étaient déroulées dans un climat tendu, l'acteur n'ayant pas adhéré à la vision du metteur en scène, si différente des pièces habituellement proposées aux comédiens de l'Ensemble. Les deux représentations s'étaient toutefois bien déroulées, et malgré le départ de quelques spectateurs, la majeure partie était restée, quelques personnes avaient même ri, comprenant l'humour décalé.

Guerre avec Maximilian Brauer

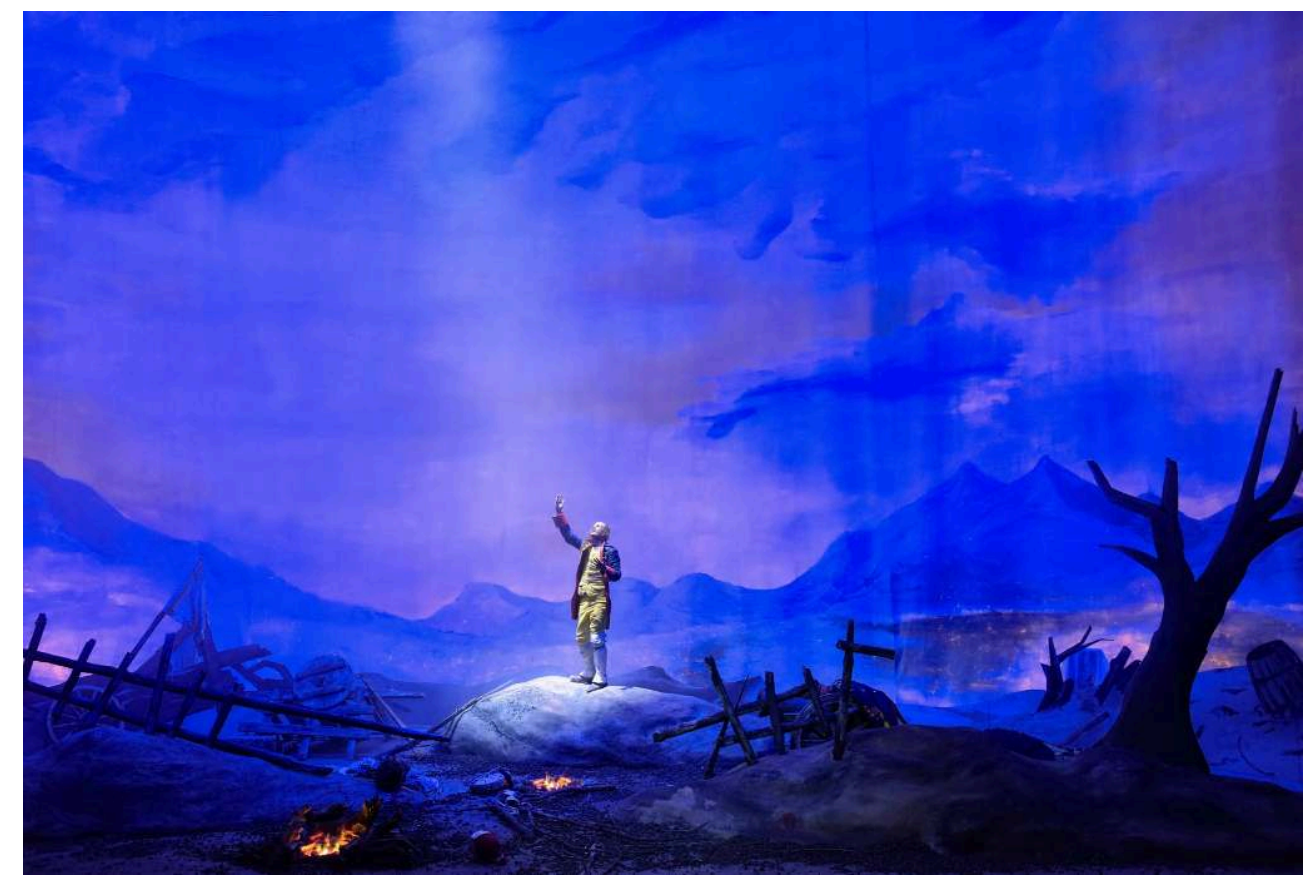

(c) Luhring Augustine, New York \& i8 Gallery, Reykjavik. Photo : Thomas Aurin.

Les jeux de lumière animaient la toile peinte d'innombrables mouvements, en fonction de la nuit qui tombait derrière le soldat mourant. Le moment fatidique, habituellement si théâtral, se trouvait grippé, anti-spectaculaire, et l'agonie, confrontée à sa réalité, très différente d'un couperet, s'étirait. Devant un spectacle si grandiloquent joué frontalement, encadré d'un lourd rideau rouge dévoilant toute son illusion, le public pouvait difficilement ressentir la moindre empathie pour le soldat. L'unique survivant de la bataille n'était pas un héros, mort d'un coup d'un seul, au cœur du combat, mais un pantin ridicule qui se débattait longuement avant de succomber, mettant fin au spectacle. 

Salon cru: un jeu cru, ne fut pas aisé pour Ragnar Kjartansson. Le concept qu'il avait élaboré autour d'un texte de la poète canadienne Anne Carson rebutait. Finalement, Bernhard Schütz et Kathrin Angerer, deux acteurs reconnus, habituels de la Volksbühne, acceptèrent. Dans un décor de salon bourgeois cliché, deux personnages, Lavinia Molson-Beck et Lord Lesley Updown, échangeaient des répliques convenues sur la guerre au Sierra Leone et les remarques idiotes des autres abonnés du studio Fitness (« Dis, penses-tu que les gens croient que les choses qu'ils voient à la télé ne se passent que là-bas ${ }^{20}$ ?»). Leur servante passait. Puis un quatrième personnage surgissait en haut de l'escalier à cour, John James Jélicho III. Ce dernier les saluait, chantait un refrain en s'accompagnant au piano, puis disparaissait, laissant le couple de nouveau seul. La scène d'une dizaine de minutes devait se répéter pendant plus de quatre heures, "au maximum cinq» annonçait le programme, selon l'endurance des comédiens, de $19 \mathrm{~h}$ à minuit. Comme aucun acteur n'avait été trouvé pour jouer l'ami qui venait saluer, Bernhard Schütz proposa que Ragnar Kjartansson endosse le rôle. L'artiste plasticien accepta donc de jouer Jélicho, personnage secondaire, sur lequel le spectateur n'apprenait pas grand-chose, si ce n'est son caractère détendu, souligné par sa chemise hawaïenne. 
Salon Cru : un jeu cru (de gauche à droite : Bernhard Schütz, Kathrin Angerer, Ragnar Kjartansson).

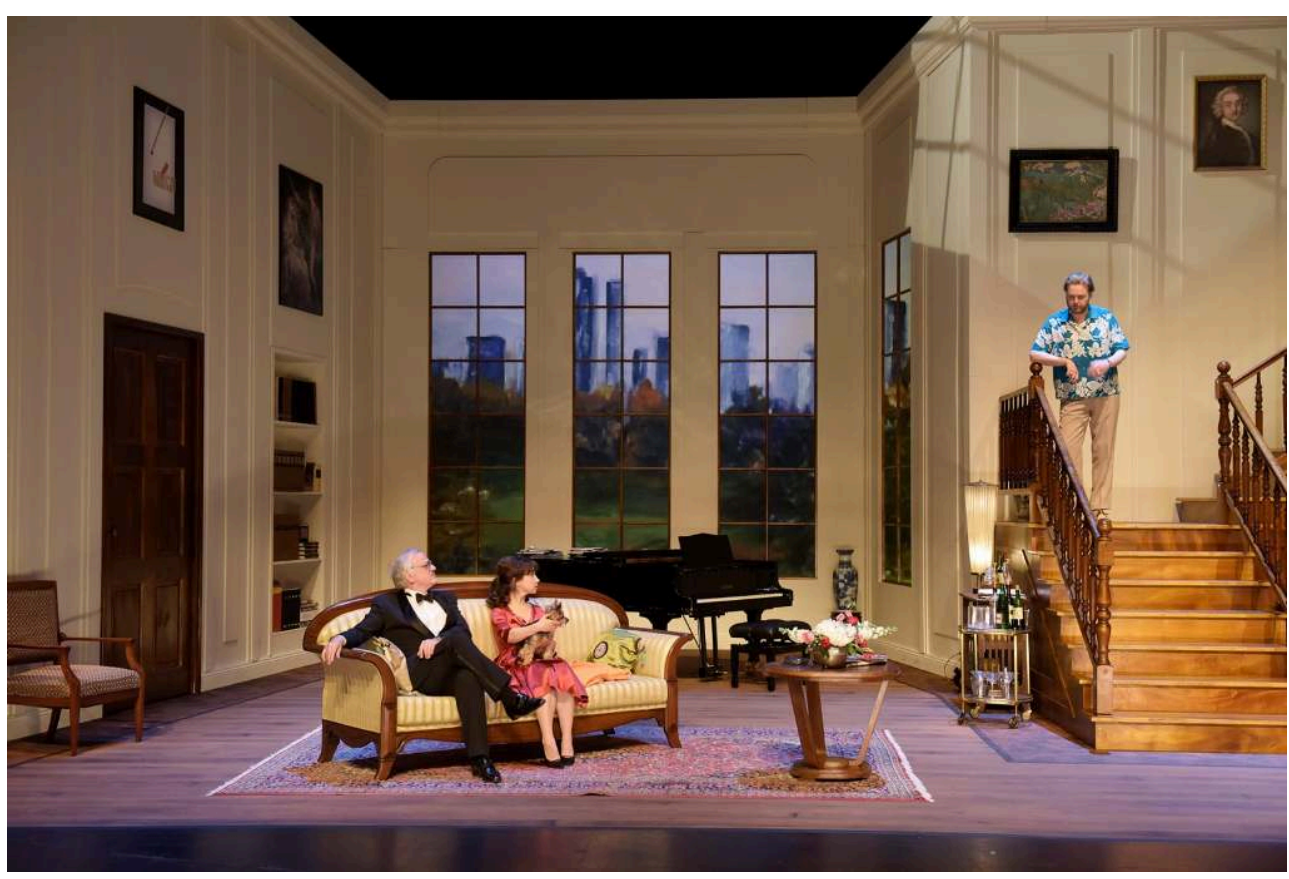

(c) Luhring Augustine, New York \& i8 Gallery, Reykjavik. Photo : Thomas Aurin.

Alors que son spectacle reposait sur le rater du dialogue dans une situation bloquée, Ragnar Kjartansson fut pris à son piège, signant, pour lui du moins, l'échec de son dispositif. En effet, au début de la boucle, les deux acteurs virtuoses, au lieu de répéter à l'identique les répliques de leur dialogue, trouvaient à chaque reprise des situations de jeu différentes. Ils excellèrent en improvisant des situations inattendues, tandis que, déconcerté, Ragnar Kjartansson participait malgré lui au comique de la pièce. Une fois, Bernhard Schütz, ouvrant le dialogue, commença à interpréter un agent secret venu voler des objets dans le salon, forçant Kathrin Angerer à s'adapter au jeu scénique. Une autre fois, tous deux entamèrent la scène pressés l'un contre l'autre, dialoguant sur un ton de séduction poussé.

À chacune de ses entrées en scène, Ragnar Kjartansson devait affronter une nouvelle situation, qu'il découvrait devant les spectateurs. Même si les répliques étaient fixes, la difficulté du texte en allemand, une langue qu'il ne maîtrisait pas bien, redoublait sa maladresse. Dans la salle, le public jubilait, les improvisations étant de plus en plus osées en fonction de l'épuisement progressif des acteurs et de leurs trouvailles de plus en plus ingénieuses après beaucoup de possibilités épuisées au plateau. Profitant de la présence du metteur en scène parmi eux, les comédiens lui prouvaient en direct combien la tâche était pénible mais comment, malgré tout, ils parvenaient à sublimer le rater prévu et le transformer en réussite. De fait, les variations, d'une fois sur l'autre, étaient passionnantes, tant à jouer qu'à regarder. Le rater se muait donc en exercice théâtral réussi ; d'un autre côté, il mettait en échec la prestation d'acteur et le projet initial de Ragnar Kjartansson.

Utilisant la trame imposée, les comédiens dévoilaient les rouages du cadre bloqué par la liberté qu'ils y développaient. Un journaliste jugea que la pièce illustrait le pouvoir de la mise en scène, en offrant une variété de situations à partir d'un même texte ${ }^{21}$. Ici, la dramaturgie du rater garantissait le succès, transcendée par le présent théâtral. Alors 
que la Volksbühne était connue pour adapter très librement les auteurs qu'elle mettait en scène, le texte était cette fois-ci exceptionnellement respecté mais les acteurs revendiquaient leur liberté sur tout le reste.

Les spectateurs, libres d'entrer et de sortir de la salle de $19 \mathrm{~h}$ à $23 \mathrm{~h}$, ne pouvaient plus la quitter à partir de $23 \mathrm{~h}$, restant vivre avec les comédiens la dernière heure de jeu avant la délivrance, à minuit précisément. Alors, le rideau se fermait, brutalement, au milieu de la boucle, sur les comédiens en train de jouer. La fin de la pièce signifiée par une simple convention théâtrale renforçait l'aspect factice du spectacle, en contraste avec l'expérience éreintante vécue par les comédiens. Comme pour Guerre, en tournant en dérision les efforts humains anéantis par le dispositif enrayé, la dramaturgie du rater avait le dernier mot.

\section{Réception de Salon cru : un jeu cru et Bonjour}

Ce dernier spectacle, mis en perspective avec Bonjour, permet de réfléchir à la réception d'un type de performance attachée à la dramaturgie du rater en France et en Allemagne. Tandis qu'au théâtre de la Volksbühne, le public s'était amusé de l'échec de la représentation, et que les comédiens avaient joué de la complicité du public les soutenant dans l'épreuve, en France, le rater était resté à sa place au musée, intriguant le public en tant que tel. L'exposition invitait à respecter attentivement un objet étrange proposé comme un cliché photographique ou peint, animé quelques minutes.

Le public de la Volksbühne, lui, était préparé à vivre une expérimentation proposée par une scène d'avant-garde, par des acteurs connus se prêtant à la déstabilisation d'un exercice de style incongru, mais, en somme, assez codifié. Les spectateurs berlinois étaient invités, dans l'espace-temps de la représentation, à réagir à la dramaturgie du rater, par le rire ou en quittant la salle; ceux qui restaient sentaient qu'il s'agissait d'un moment intime avec de grands acteurs mis en difficulté. Les visiteurs du Palais de Tokyo adhéraient au contraire à la dramaturgie du rater, en explorant individuellement, parfois sur plusieurs boucles, une frustration croissante. Le spectacle du rater, illustratif dans Bonjour, devenait un exercice de style contre la monotonie dans le dernier spectacle. La place des performeurs variait en fonction: au Palais de Tokyo, ils effaçaient leur singularité, à la Volksbühne, leur personnalité déterminait les inventions scéniques.

\section{Réflexions sur la répétition du rater}

Pour chacune de ces performances, Ragnar Kjartansson ne mettait pas l'accent sur la fable mais sur l'action représentée en elle-même et pour elle-même. Pour reprendre les catégories de Michael Kirby, «le processus réel de la 'performance' rempla[çait] le 'mimetic acting'", et «ne défini[ssait] plus seulement un 'extrême', mais une dimension primordiale de la nouvelle réalité du théâtre ${ }^{22}$ ». Le rater, du fait de sa répétition, permettait de constater l'échec d'une action jamais aboutie; c'est dans cet inachèvement permanent que résidait le tragique de l'action humaine. La chercheuse en arts du spectacle Laure Fernandez analysait :

Des pièces, des drames, des histoires jouées, Ragnar Kjartansson se soucie donc peu. Ce qui le marque, l'inspire, ce sont les coulisses, l'exercice de la mise en travail des acteurs, les récits accompagnant les créations, avec tout ce que cet envers de décor 
contient de force poétique - répétitions, ratages, jeux de machinerie, images et sons de ces moments où se construit, encore et encore, quelque chose qui n'advient pas, qui n'est pas tout à fait prêt à advenir ; ces moments où l'action n'est pas encore un élément de construction d'une fable, n'est pas encore la partie d'un ensemble, mais un acte isolé qu'il s'agit de dessiner et de préciser; où l'illusion est imparfaite et cette imperfection, fascinante ${ }^{23}$.

43

Derrière la répétition des poncifs du quotidien, Ragnar Kjartansson accède à une fresque des temps contemporains, comme Roland Barthes dans ses Mythologies:

Écrits mois après mois, ces essais ne prétendent pas à un développement organique : leur lien est d'insistance, de répétition. Car je ne sais, si, comme dit le proverbe, les choses répétées plaisent, mais je crois que du moins elles signifient ${ }^{24}$.

En revisitant trois situations clichées dans les performances analysées, Ragnar Kjartansson insistait sur des images devenues des lieux communs de notre société, dévoilant, sous l'envers d'un décor, leurs sens cachés. Il semblait aider à comprendre dans quelle mesure " la réalité ne peut être franchie que soulevée ${ }^{25}$ ", selon la formule de René Char.

Dans une société noyée sous les images stéréotypées, l'artiste islandais en sélectionne certaines, les tourne en dérision par des phénomènes de blocage, et les réinvestit de leur force poétique. Par la théâtralisation de clichés qu'il affectionne et dont il montre l'envers, il démontre autant qu'il dénonce leur force de séduction. En «nostalgique tourné vers l'avant » (« nostalgic in a forward-looking way $\left.{ }^{26} »\right)$, Ragnar Kjartansson livre une véritable mythologie des représentations qui nous ont précédés, dans lesquelles nous nous inscrivons et qui nous survivront.

BIBLIOGRAPHIE

\section{Ouvrages sur le théâtre et la performance}

BARTHES, Roland, Mythologies, Roland Barthes, Paris, Éditions du Seuil, 1957.

BRECHT, Bertolt, Petit organon pour le théâtre, Paris, L'Arche, 1963.

DELEUZE, Gilles, Différence et Répétition, Paris, PUF, 1968.

FERNANDEZ, Laure, « Une fois, encore », catalogue de l'exposition « Seul celui qui connaît le désir, Ragnar Kjartansson », Palais de Tokyo, Dijon, Les Presses du Réel, 2015.

FISCHER-LICHTE, Erika, Ästhetik des Performativen, Francfort-sur-le-Main, Suhrkamp, 2004.

-, Performativität, eine Einführung, Bielefeld, Transcript, 2012.

KJARTANSSON, Ragnar et FRONSACQ, Julien, Entretien, catalogue de l'exposition « Seul celui qui connaît le désir, Ragnar Kjartansson », Palais de Tokyo, Dijon, Les Presses du Réel, 2015.

LEHMANN, Hans-Thies, Le Théâtre postdramatique, traduit de l'allemand par Philippe-Henri Ledru, Paris, L'Arche, 2002 (Postdramatisches Theater, Francfort-sur-le-Main, Verlag der Autoren, 1999).

NIETZSCHE, Friedrich, Le Gai Savoir (1882), traduction de Patrick Wotling, Paris, GF Flammarion, 2007. 
-, Ecce Homo (1908), traduction d'Eric Blondel, Paris, GF Flammarion, 1992.

\section{Articles de presse en ligne}

CUMMING Laura, « Ragnar Kjartansson review - a brilliant fusion of humour and sorrow », theguardian.com, 17 juillet 2016, URL : <https://www.theguardian.com/artanddesign/2016/jul/ 17/ragnar-kjartansson-barbican-review> [Site consulté le 19 octobre 2020].

EHLERT Sascha, « Menschen? Unbedeutend! », nachtkritik.de, 11 mai 2016, URL : <https:// www.nachtkritik.de/index.php?option=com_content\&view=article\&id=12258:krieg-derperformance-kuenstler-ragnar-kjartansson-ueberlaesst-seinen-hauptdarsteller-maximilianbrauer-wortlos-dem-tod\&catid=38:die-nachtkritik-k\&Itemid=40> [Site consulté le 22 octobre 2020].

GOPNIK, Blake, «In His Hands, a Love Song Becomes a Requiem for Plague Times », nytimes.com, 18 septembre 2020, URL : <https://www.nytimes.com/2020/09/18/arts/design/ragnarkjartansson-milan.html> [Site consulté le 19 octobre 2020].

NEEDHAM, Alex, theguardian.com, « The maddest house party ever - Ragnar Kjartansson on making The Visitors ", theguardian.com, 17 septembre 2019, URL : <https:// www.theguardian.com/artanddesign/2019/sep/17/ragnar-kjartansson-the-visitors-best-artwork-21st-century> [Site consulté le 19 octobre 2020].

RAKOW, Christian, « Und täglich blüht das Blümchenhemd », nachtkritik.de, 06 avril 2017, URL :

$<$ https://www.nachtkritik.de/index.php?option=com_content\&view=article\&id=13838:raw-salonein-rohspiel-ragnar-kjartansson-loopt-an-der-berliner-volksbuehne-einen-10-minuten-text-vonanne-carson-auf-castorf-sche-laenge\&catid=38\&Itemid=40> [Site consulté le 22 octobre 2020].

TOMKINS, Calvin, newyorker.com, 11 avril 2016, URL : <https://www.newyorker.com/magazine/ 2016/04/11/ragnar-kjartansson-on-repeat> [Site consulté le 19 octobre 2020].

Site Anthea Missy, 10 novembre 2018, URL : <https://www.antheamissy.com/in-iceland-itscommon-to-listen-to-american-pop-songs-and-laugh-about-how-misogynist-they-are-ragnarkjartansson-on-his-new-musical-work/> [Site consulté le 19 octobre 2020].

Site de la Volksbühne, URL : https://volksbuehne.adk.de, <https://volksbuehne.adk.de/praxis/ raw_salon/index.html> [Site consulté le 22 octobre 2020].

\section{Euvres}

RAGNAR KJARTANSSON Bonjour, 2015, performé comme une partie de Seul celui qui connaît le désir au Palais de Tokyo, Paris, du 21 octobre 2015 au 10 janvier 2016, chaque jour pendant 12 heures. Avec l'aimable autorisation de l'artiste, Luhring Augustine, New York \& i8 Gallery, Reykjavik. Photo : Justine Emard. Vidéo : Charles Mignon.

RAGNAR KJARTANSSON Krieg, 2016, opéra en un acte joué à la Volksbühne am Rosa-LuxemburgPlatz. Musique de Kjartan Sveinsson. Avec l'aimable autorisation de l'artiste, Luhring Augustine, New York \& i8 Gallery, Reykjavik. Photo : Thomas Aurin.

RAGNAR KJARTANSSON Raw Salon: Ein Rohspiel, 2017, pièce de théâtre jouée à la Volksbühne am Rosa-Luxemburg-Platz. Texte : Anne Carson. Musique : Kjartan Sveinsson. Avec l'aimable autorisation de l'artiste, Luhring Augustine, New York \& i8 Gallery, Reykjavik. Photo : Thomas Aurin. 


\section{NOTES}

1. D'après Le petit Robert de la langue française, édition 2020.

2. Torstov dans La Formation de l'acteur de Stanislavski ou encore Constance Dumas, jouée par Bulle Ogier, dans le film La Bande des quatre (1988) de Jacques Rivette, préviennent leurs élèves contre les clichés. On retient la leçon marquante de cette deuxième figure de pédagogue : «la démolition, c'est avec ça que vous avez à faire tout le temps. La démolition et le doute, c'est avec ça que vous devez construire, créer, inventer ». Elle invite ainsi ses apprenties comédiennes à se confronter à l'intériorité et aux questions fondamentales de chaque rôle. (Citation de La Bande des quatre de Jacques Rivette, disponible sur le site de la Cinémathèque, cinematheque.fr, URL: <https://www.cinematheque.fr/seance/26589.html> [Site consulté le 15 novembre 2020]).

3. Il n'y a d'article "cliché » ni dans le Dictionnaire du théâtre de Patrice Pavis, ni dans le Dictionnaire encyclopédique du Théâtre de Michel Corvin.

4. Le Théâtre pour lui-même, cité dans Erika Fischer-Lichte, Performativität, eine Einführung, Bielefeld, Transcript, 2012. Notre traduction. Toutes les traductions suivantes sont de notre fait.

5. Laure Fernandez, "Une fois, encore», catalogue de l'exposition «Seul celui qui connaît le désir, Ragnar Kjartansson », Palais de Tokyo, Dijon, Les Presses du Réel, 2015.

6. Erika Fischer-Lichte, Ästhetik des Performativen, Francfort-sur-le-Main, Suhrkamp, 2004.

7. Comme je l'explique par la suite, je m'appuierai, pour cet aspect, sur mon expérience personnelle en tant que performeuse dans l'œuvre du Palais de Tokyo.

8. Site de la Volksbühne, URL : https://volksbuehne.adk.de, <https://volksbuehne.adk.de/praxis/ raw_salon/index.html>, Ragnar Kjartansson: "With repetition, narrative things like songs, concerts, or operas can lose their traditional form and become static - but vibrant, like paintings or sculptures, I often look at my performances as sculptures and the videos as paintings ", [Site consulté le 22 octobre 2020].

9. Ragnar Kjartansson, dans l'entretien avec Julien Fronsacq, catalogue de l'exposition «Seul celui qui connaît le désir », op. cit.

10. Ibidem.

11. Le budget conséquent de la performance a été pris en charge par le Palais de Tokyo et le Festival d'Automne à Paris.

12. Heiner Müller, cité dans l'appel à contribution de ce numéro 9 de la revue Agôn, ibid. : « Le théâtre s'engendre seulement au point d'intersection entre angoisse et géométrie ", traduction de Jean Jourdheuil et Jean-Louis Besson, in Frictions n²4, hiver 2014-2015, publié auparavant dans un programme du Théâtre de la Ville (Paris), novembre-décembre 2009, p. 13.

13. Ibid., p. 61.

14. Entretien avec Julien Fronsacq, catalogue de l'exposition «Seul celui qui connaît le désir, Ragnar Kjartansson », op. cit., p. 62.

15. Schlingensief, acceptant cette règle, se lança dans plusieurs mises en scène osées, risquant de déplaire au public. En 1998, il affirma la primauté de l'échec dans son travail lorsqu'il fonda un vrai parti politique, "Chance 2000", "Parti de la dernière chance" ("Chance 2000", "Partei der letzten Chance"), dont le slogan était "Échouer est une chance". Conçu pour représenter tous les marginalisés de la société, le parti loufoque brigua réellement les élections parlementaires du Bundestag.

16. Tanja Bogusz, Institution und Utopie, Ost-West Transformationen an der Berliner Volksbühne, Bielefeld, transcript Verlag, 2007, p. 207 : « Frank Castorf [...]: « Ick hab Schlingensief jesagt, ween es kein Erfolg wird, en halben Flop kann ick mir nicht leisten. Wenn's ein richtiger Flop wird, dann isses wieder jut. Schlingensief sagt: ja, kann ich garantieren ". 
17. Ce débat sur le théâtre postdramatique est régulièrement relancé. Pour comprendre l'écho que prend ce concept, à travers les décennies passées, en France et en Allemagne, on peut se référer à l'article suivant : Wolfgang Asholt, Université Humboldt (Berlin), "Théâtre post-dramatique et/ou Storytelling? », Fabula / Les colloques, « Pratiques contre-narratives à l'ère du storytelling. Littérature, audiovisuel, performances », URL : http://www.fabula.org/colloques/document6075.php, page consultée le 20 août 2021.

18. Hans-Thies Lehmann, Le Théâtre postdramatique, traduit de l'allemand par Philippe-Henri Ledru, Paris, L'Arche, 2002 (Postdramatisches Theater, Francfort-sur-le-Main, Verlag der Autoren, 1999), p. 158.

19. Ibid., p. 160.

20. Christian Rakow, article Nachtkritik « Und täglich blüht das Blümchenhemd », nachtkritik.de, 06 avril 2017, URL : https://www.nachtkritik.de/index.php? option=com_content\&view=article\&id=13838:raw-salon-ein-rohspiel-ragnar-kjartansson-looptan-der-berliner-volksbuehne-einen-10-minuten-text-von-anne-carson-auf-castorf-sche-

laenge\&catid=38\&Itemid $=40:$ « Sag mal, denkst du , die meisten Menschen glauben, die Dinge, die sie im TV sehen, passieren nur dort? ", [Site consulté le 22 octobre 2020]. Le texte d'Anne Carson fut traduit par Angela Rosenberg.

21. Ibidem.

22. Hans-Thies Lehmann, Le Théâtre postdramatique, op.cit., p. 49.

23. Laure Fernandez, «Une fois, encore», catalogue de l'exposition «Seul celui qui connaît le désir, Ragnar Kjartansson », op. cit., p. 21.

24. Roland Barthes, Mythologies, Paris, Éditions du Seuil, 1957, p. 10.

25. Poème «Les dentelles de Montmirail» cité par Laure Fernandez à propos du travail de l'artiste dans le catalogue de l'exposition "Seul celui qui connaît le désir, Ragnar Kjartansson ", op. cit., p. 20.

26. Laure Fernandez citant Kjartansson, catalogue de l'exposition « Seul celui qui connaît le désir, Ragnar Kjartansson », ibid., p. 22.

\section{RÉSUMÉS}

Grandi dans un univers de spectacles et de représentations, l'artiste plasticien et performeur islandais Ragnar Kjartansson est fasciné par les clichés. Entre l'envers et l'endroit du décor, il ne saurait choisir. Dans ses œuvres plastiques et performatives, il se saisit de clichés esthétiques pour montrer leur envers, des processus grippés d'actions bloquées, répétées en boucle pendant plusieurs heures. L'article étudie la dramaturgie du rater dans trois de ses spectacles entre la France et l'Allemagne : d'une part Bonjour (2015) au Palais de Tokyo à Paris, d'autre part Guerre (Krieg, 2016) et Salon cru : un jeu cru, (Raw Salon: ein Rohspiel, 2017) à la Volksbühne de Berlin. Le positionnement des acteurs au sein des performances ainsi que la réception d'un pays à l'autre est éclairé en fonction des dispositifs et des lieux d'accueil des œuvres. 
INDEX

Mots-clés : Kjartansson (Ragnar), cliché, envers du décor, Palais de Tokyo, Volksbühne, répétition, jeu de l'acteur.

\section{AUTEUR}

\section{HÉLISENNE LESTRINGANT}

Hélisenne Lestringant, ancienne élève de l'École normale supérieure de Lyon, agrégée d'allemand, est doctorante en Études théâtrales sous la direction de Marielle Silhouette à l'Université Paris Nanterre, en cotutelle avec l'université d'Hildesheim en Allemagne, sous la direction d'Annemarie Matzke. Elle rédige une thèse sur l'œuvre du réalisateur, performeur et metteur en scène contemporain Christoph Schlingensief, et est chargée de cours en Études théâtrales à l'Université Paris Nanterre et en Études germaniques à l'Université Paris 8. À l'occasion de son Master 2 consacré aux collectifs alternatifs de la « freie Szene » à Berlin, sous la direction de Jean-Louis Besson, elle pénètre la scène allemande contemporaine. Alliant théorie et pratique théâtrales en France et en Allemagne, elle effectue des stages à la Volksbühne de Berlin sous la direction de Frank Castorf et au Berliner Ensemble. Elle est aussi à l'origine de performances dans des lieux insolites : dans des appartements à Paris avec le collectif 7x7 qu'elle a cofondé avec A. Anne-Braun et J. Riedler, à l'ancienne fonderie de métal de Lichtenberg ou dans la communauté d'habitation Pa 58 de Wedding Berlin. Elle est membre de la compagnie de théâtre itinérante « Les Nuits blondes » et dirige le collectif franco-allemand de performances site-specific « Visite ma tente». 